\title{
RING domain-deficient BRCA1 promotes PARP inhibitor and platinum resistance
}

\author{
Yifan Wang, ${ }^{1}$ John J. Krais, ${ }^{1}$ Andrea J. Bernhardy, ${ }^{1}$ Emmanuelle Nicolas, ${ }^{2}$ Kathy Q. Cai, ${ }^{1}$ Maria I. Harrell, ${ }^{3}$ Hyoung H. Kim, ${ }^{4}$ \\ Erin George, ${ }^{4}$ Elizabeth M. Swisher, ${ }^{3}$ Fiona Simpkins, ${ }^{4}$ and Neil Johnson ${ }^{1}$ \\ ${ }^{1}$ Molecular Therapeutics Program and ${ }^{2}$ Cancer Biology Program, Fox Chase Cancer Center, Philadelphia, Pennsylvania, USA. ${ }^{3}$ Department of Obstetrics and Cynecology, University of Washington, \\ Seattle, Washington, USA. ${ }^{4}$ Department of Obstetrics and Gynecology, University of Pennsylvania, Philadelphia, Pennsylvania, USA.
}

\begin{abstract}
Patients with cancers that harbor breast cancer 1 (BRCA1) mutations initially respond well to platinum and poly(ADP-ribose) polymerase inhibitor (PARPi) therapy; however, resistance invariably arises in these patients and is a major clinical problem. The BRCA 185delAC allele is a common inherited mutation located close to the protein translation start site that is thought to produce a shortened, nonfunctional peptide. In this study, we investigated the mechanisms that lead to PARPi and platinum resistance in the SUM1315M02 breast cancer cell line, which harbors a hemizygous BRCA1 ${ }^{185 d e l A C}$ mutation. SUM1315MO2 cells were initially sensitive to PARPi and cisplatin but readily acquired resistance. PARPi- and cisplatin-resistant clones did not harbor secondary reversion mutations; rather, PARPi and platinum resistance required increased expression of a really interesting gene (RING) domain-deficient BRCA1 protein (Rdd-BRCA1). Initiation of translation occurred downstream of the frameshift mutation, probably at the BRCA1-Met-297 codon. In contrast to full-length BRCA1, Rdd-BRCA1 did not require BRCA1-associated RING domain 1 (BARD1) interaction for stability. Functionally, Rdd-BRCA1 formed irradiation-induced foci and supported RAD51 foci formation. Ectopic overexpression of Rdd-BRCA1 promoted partial PARPi and cisplatin resistance. Furthermore, Rdd-BRCA1 protein expression was detected in recurrent carcinomas from patients who carried germline BRCA185delAC mutations. Taken together, these results indicate that RING-deficient BRCA1 proteins are hypomorphic and capable of contributing to PARPi and platinum resistance when expressed at high levels.
\end{abstract}

\section{Introduction}

Germline breast cancer 1 (BRCA1) mutations dramatically increase a carrier's lifetime risk of developing breast and ovarian cancer (1-3). Pathogenic BRCA1-mutant alleles fail to generate protein products with tumor-suppressor activity, and cancer initiation usually results from loss of heterozygosity $(\mathrm{LOH})$ affecting the BRCA1 WT allele (3-5). The BRCA1 protein is an 1863-aa peptide containing several highly conserved domains. The N-terminal contains a RING domain that is commonly found in E3 ubiquitin ligases and is required for heterodimerization with BRCA1-associated RING domain 1 (BARD1). BRCA1 interacts with partner and localizer of BRCA2 (PALB2) through a coiled-coil region that facilitates the formation of a BRCA1-PALB2-BRCA2-RAD51 complex. The BRCA1 C-terminus domains (BRCT) bind phosphorylated proteins such as CtIP and abraxas (5-7).

The BRCA1 protein is necessary for efficient homologous recombination (HR) DNA repair (8), and mutations in the $B R C A 1$ gene that render the protein product dysfunctional result in cellular sensitivity to poly(ADP-ribose) polymerase inhibitors (PARPi) $(9,10)$, as well as to DNA-damaging platinum agents $(8,11,12)$. Ovarian and breast cancer patients who harbor

\section{Related Commentary: p. 2802}

Conflict of interest: The authors have declared that no conflict of interest exists. Submitted: February 12, 2016; Accepted: May 9, 2016.

Reference information: J Clin Invest. 2016;126(8):3145-3157. doi:10.1172/JCI87033.
BRCA1 mutations respond well to initial platinum-based therapy (13-15), and several PARPi are currently under advanced-stage clinical investigation, as they have demonstrated clinical activity in patients with $B R C A$ mutations (15-17). Despite survival improvements, resistance to both PARPi and platinum therapy invariably arises and is a major clinical problem (18-21). Secondary $B R C A 1$ and $B R C A 2$ gene-reversion mutations that restore the reading frame represent the most well-validated mechanism of platinum resistance (22-25). Several mechanisms of PARPi resistance have been described in Brca1-mutated mouse mammary tumors, including activation of P-glycoprotein (26) and loss of 53BP1 expression (27-29).

Cellular mechanisms that result in increased levels of truncated, but hypomorphic, BRCA1 proteins have been shown to result in both PARPi and platinum resistance. We previously demonstrated that cells harboring a BRCT domain-truncating mutation were able to develop resistance to PARPi and cisplatin through overexpression of a BRCT domain-deficient BRCA1 protein (30). Despite lacking the BRCT domain, the retention of all other critical regions, including the PALB2-interacting domain, facilitated RAD51 loading and therapy resistance. Similarly, when the Brca1 ${ }^{\mathrm{C} 61 G}$ allele was introduced into a conditional mouse model of BRCA1-associated breast cancer, tumors formed at a rate similar to that seen in Brcal-deficient mice. However, tumors carrying Brca1 ${ }^{\mathrm{C} 61 G}$ responded poorly to platinum and PARPi (31). Intriguingly, a similar study that introduced the $B r c a 1^{I 26 A}$ synthetic missense variant that reduces 
A
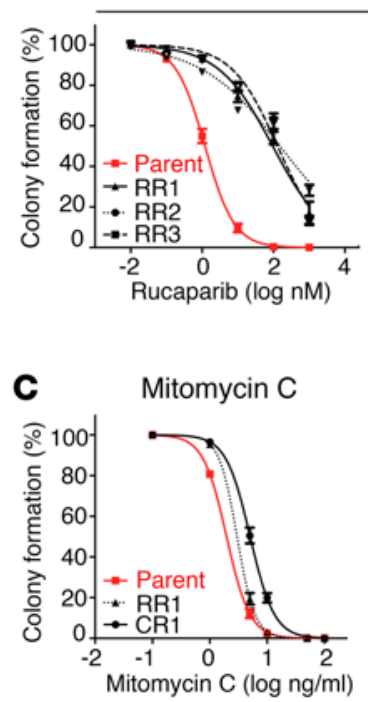

PARPi-derived
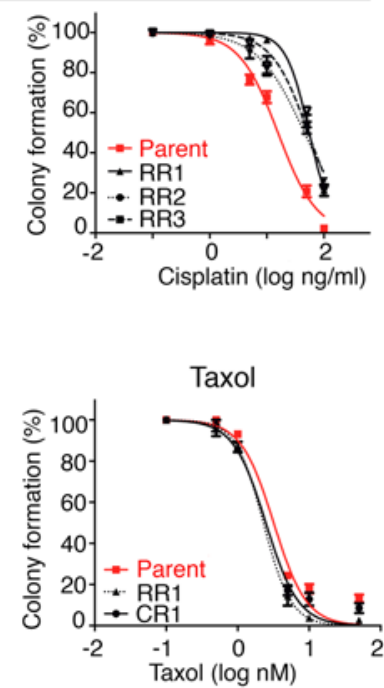

B

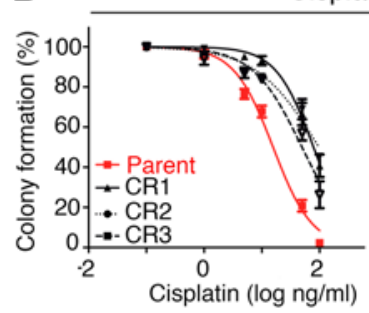

Cisplatin-derived

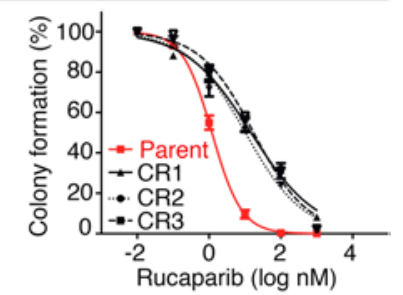

D

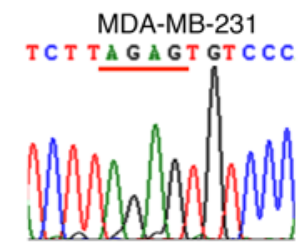

SUM1315 RR1

T C T T A G T G T C C C

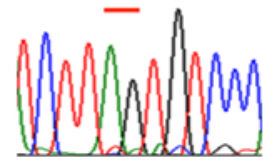

SUM1315 Parental

T C T T A G T T C C C

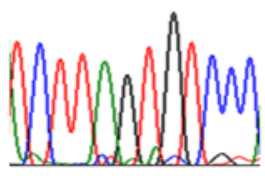

SUM1315 CR1

T C T T $A$ G T T C C C

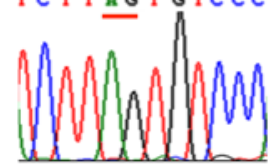

Figure 1. SUM1315M02 PARPi- and cisplatin-resistant cells do not harbor reversion mutations. (A) SUM1315MO2 parental cells and RR1-3 cells were measured for primary resistance to rucaparib and cross-resistance to cisplatin. (B) SUM1315M02 parental cells and CR1-3 cells were measured for primary resistance to cisplatin and cross-resistance to rucaparib. (C) SUM1315MO2 parental cells and RR1 and CR1 cells were assessed for mitomycin C and taxol sensitivity. Colony formation assays were employed throughout and three independent experiments were performed. (D) $B R C A 1$ introns and exons from parental cells and resistant clones were subjected to Sanger sequencing; representative BRCA1 electropherograms are shown for parental cells and 6 resistant clones that were sequenced.

E3-ubiquitin ligase activity, but does not prevent BRCA1-BARD1 assembly, did not impact BRCA1 tumor-suppressor activity, and mice were resistant to tumor formation (32).

The BRCA1 $1^{185 d e l A G}$ allele is a common founder mutation, prevalent in approximately $1 \%$ of the Ashkenazi Jewish population and predisposes carriers to early-onset breast and ovarian cancer (33, $34)$. Here, we report that $B R C A 1^{185 d e l A G}$ frameshift mutation-carrying cell lines and tumors are capable of producing RING domaindeficient BRCA1 proteins (Rdd-BRCA1) that facilitate RAD51 foci formation and PARPi and cisplatin resistance.

\section{Results}

SUM1315MO2 PARPi- and cisplatin-resistant cells do not acquire secondary reversion mutations. The SUM1315MO2 cell line was derived from a skin metastasis of a female patient with invasive ductal carcinoma. SUM1315MO2 cells have LOH at the BRCA1 locus and harbor the common pathogenic $B R C A 1^{185 d e l A G}$ allele $(35,36)$. To understand the impact of the $B R C A 1^{185 d e l A G}$ allele on drug resistance, we cultured SUM1315MO2 cells in the presence of increasing concentrations of either the PARPi rucaparib or cisplatin, until 3 individual resistant clones were derived for each agent. SUM1315MO2 cells rapidly acquired PARPi and cisplatin resistance, and resistant clones were derived within 1 to 2 months from initial exposure.

We confirmed by colony formation assay that the clones demonstrated drug resistance. Rucaparib-resistant clones 1-3 (RR1-3) were 84- $(P=0.024), 128$ - $(P=0.008)$, and $110-$ fold $(P=0.01)$ more resistant to rucaparib treatment than were SUM1315MO2 parental cells. Additionally, RR1-3 were 3.6- $(P<0.001), 3-(P=0.002)$, and 3.5-fold
$(P<0.001)$ more resistant to cisplatin compared with parental cells (Figure 1A). SUM1315MO2 cells cultured in the presence of cisplatin were also measured for cisplatin and PARPi sensitivity. Cisplatinresistant clones 1-3 (CR1-3) were 5.1- $(P=0.006)$, 6.3- $(P<0.001)$, and 3.3-fold $(P=0.008)$ more resistant to cisplatin treatment and were 12- $(P=0.002), 9$ - $(P=0.021)$, and 14 -fold $(P=0.005)$ more resistant to rucaparib than were SUM1315MO2 parental cells (Figure 1B). Furthermore, RR clone 1 (RR1) and CR clone 1 (CR1) were 1.5- $(P=0.008)$ and 2.6-fold $(P=0.001)$ more resistant than parental cells to the DNA cross-linking agent mitomycin $C$, respectively. In contrast, parental, RR1 and CR1 cells demonstrated similar levels of sensitivity to the microtubule inhibitor taxol (Figure 1C).

Platinum-resistant $B R C A 1^{185 d e l A G}$ patient carcinomas have previously been shown to develop reversion mutations that restore the WT sequence (22). We sequenced the BRCA1 gene introns and exons, as well as mRNA, in parental and resistant clones and found that the original $B R C A 1^{185 d e l A G}$ mutation remained present; moreover, no additional $B R C A 1$ mutations were acquired in any of the resistant clones (Figure 1D). Because we could not detect reversion mutations in SUM1315MO2 resistant cell lines, we investigated the possibility that mutations in other DNA repair genes could be providing resistance by sequencing a panel of candidate genes using BROCA analysis (37). BROCA sequencing confirmed the presence of the known BRCA1 and TP53 mutations present in both parental and resistant clones, but there were no additional mutations in a series of genes associated with resistance, including TP53BP1 (Supplemental Table 1; supplemental material available online with this article; doi:10.1172/JCI87033DS1). 

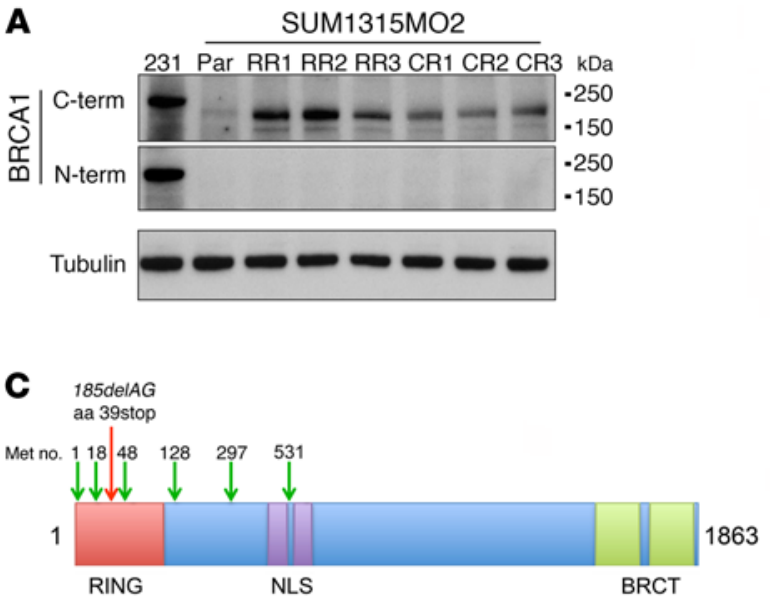
KFCMETLKLLNOKKGPSOCPLCKNDITKRSLOESTRFSOLVEELLKI CAFQLDTGLEYANSYNFAKKENNSPEHLKDEVSIIQSMETGYRNRAK RLLQSEPENPSLOETSLSVOLSNLGTVRTLRTKORIOPOKTSVYIEL GSDSSEDTVNKATYCSVGDOELLOITPQGTRDEISLDSAKKAACEF GSDSEDTVNKATYCSVGDQELLQITPQGTRDEISLDSAKKAACEF SETDVTNTEHHQPSNNDLNTTEKRAAERHPEKYQGSSVSNLHVEP CGTNTHASSLQHENSSLLLTKDRMETNVEKAEFCNKSKQPGLARSQ ENPRDTEDVPW 372

D

Endogenous Exogenous

SUM1315 MDA-MB-436

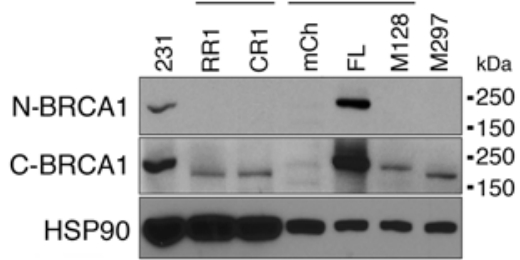

B

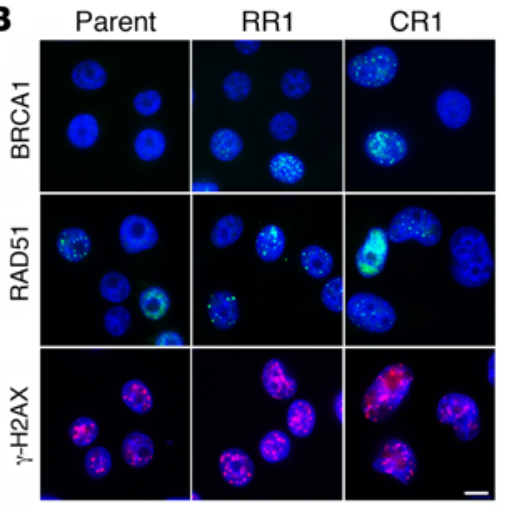

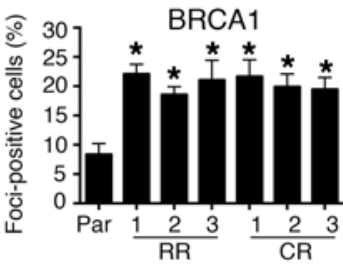

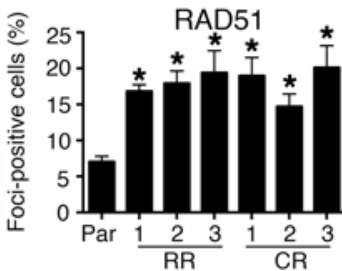

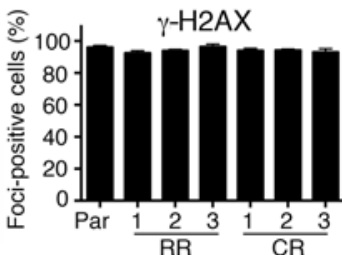

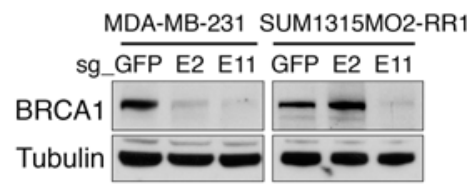

Figure 2. SUM1315M02 cells express a RING domain-deficient BRCA1 protein. (A) Cell lysates were generated from MDA-MB-231 (abbreviated as 231 in the figure), SUM1315M02 parental (Par), RR1-3, and CR1-3 cells, and BRCA1 protein was detected by Western blot analysis using C-terminal-specific (C-term) and $\mathrm{N}$-terminal-specific ( $\mathrm{N}$-term) BRCA1 Abs. Molecular weight markers are indicated. Three independent experiments were performed. (B) SUM1315M02 parental, RR1-3, and CR1-3 cells were treated with IR, and BRCA1, RAD51, $\gamma$-H2AX, and DAPI were detected by immunofluorescence (representative images are shown). Scale bar: $10 \mu \mathrm{m}$. Bar graphs show the mean and SEM percentage of cells containing more than 5 foci ( $n=3$ independent experiments. ${ }^{*} P<0.05$, 2-tailed Student's $t$ test). (C) In-frame methionine translation start sites are depicted, along with the location of the stop codon generated from the BRCA185delAC mutation (above). BRCA1 was immunoprecipitated from MDA-MB-231 and SUM1315M02 RR1 cells and subjected to mass spectrometry. BRCA1 peptides detected from MDA-MB-231 cells are highlighted in yellow and SUM1315MO2 RR1 cells in green, Met-297 is highlighted in red (below). See Supplemental Figure 1 for complete information on peptide coverage. (D) Cell lysates were collected from MDA-MB-231 and SUM1315M02 RR1 and CR1 cells, as well as from MDA-MB-436 cells that expressed exogenous mCherry (mCh), BRCA1 full-length (FL), BRCA1-Met128 (M128), or BRCA1Met-297 (M297). BRCA1 gel migration was detected by Western blot analysis using N- and C-terminal-specific Abs; HSP90 was measured as a loading control. (E) CRISPR/Cas9 targeting sg_GFP, sg_exon 2 (E2), or sg_exon 11 (E11) in MDA-MB-231 and SUM1315MO2 RR1 cells. Western blot analyses of BRCA1 levels using a C-terminal-specific Ab (see Supplemental Figure 2 for additional information). Three independent experiments were performed.

SUM1315MO2 resistant cells express a RING domain-deficient $B R C A 1$ protein. To investigate potential BRCA1 protein products generated from the BRCA1 ${ }^{185 d e l A G}$ allele in SUM1315MO2 parental and resistant clones, we measured BRCA1 protein levels by Western blotting with Abs that specifically recognize the $\mathrm{N}$ - and C-terminal regions of BRCA1. As a control, WT BRCA1 protein expressed in MDA-MB-231 cells was detectable with both $\mathrm{N}$ - and C-terminal Abs. In contrast, BRCA1 protein was undetectable in both SUM1315MO2 parental and resistant clones using the $\mathrm{N}$-terminal-specific Ab. However, the C-terminal Ab identified a band migrating slightly below full-length BRCA1 that was of low abundance in parental cells, but with elevated expression in both RR and CR clones (Figure 2A). Moreover, the BRCA1 protein detected in SUM1315MO2 cells was capable of forming $\gamma$ irradiation-induced foci (IRIF), and we readily detected both BRCA1 and
RAD51 foci in parental SUM1315MO2 cells. However, both RR and CR clones had a 2.22- to 2.64-fold $(P=0.004-0.021)$ increase in BRCA1 IRIF and a 2.1- to 2.85-fold $(P=0.0001-0.03)$ increase in RAD51 IRIF compared with parental cells, respectively. The levels of $\gamma$-H2AX, a marker for DNA damage, did not significantly differ between cell lines (Figure 2B).

We hypothesized that cells were utilizing an in-frame translation start site downstream of the $B R C A 1^{185 d e l A G}$ mutation to generate a RING domain-deficient BRCA1 protein. To identify the possible downstream translation start site, we immunoprecipitated endogenous BRCA1 from SUM1315MO2 RR1, as well as WT-expressing MDA-MB-231 cells and analyzed BRCA1 peptides by mass spectrometry. Total peptide coverage was overall greater in MDA-MB-231 cells (58\% total peptide coverage) compared with that in SUM1315MO2 RR1 cells (19\% total peptide cover- 
A IP: C-term BRCA1 SUM1315 Input -Ab $231 \overline{\mathrm{RR} 1 \mathrm{CR} 1}$

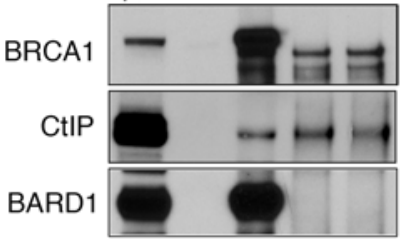

B

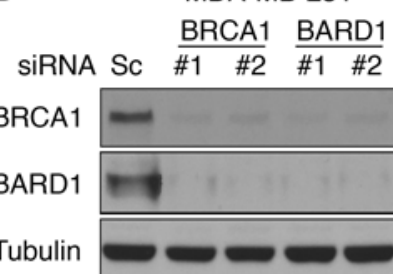

MDA-MB-436-RR1

BRCA1 BARD1

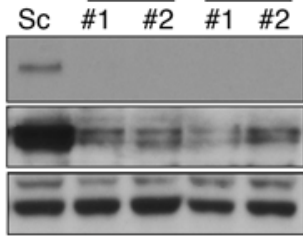

SUM1315MO2-RR1

BRCA1 BARD1

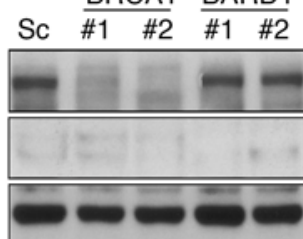

C
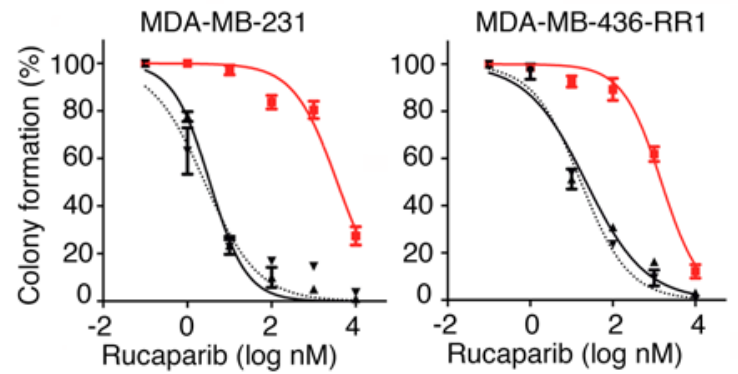

SUM1315MO2-RR1

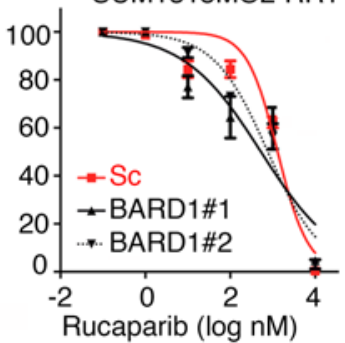

D
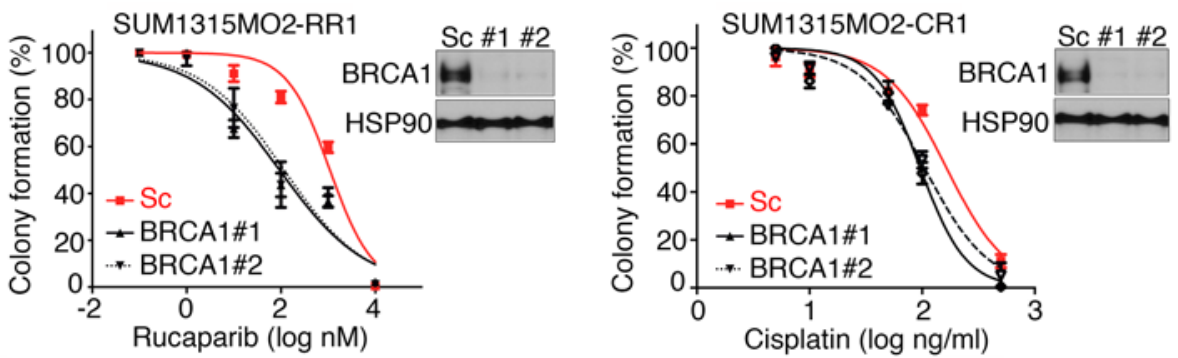

$\mathbf{E}$
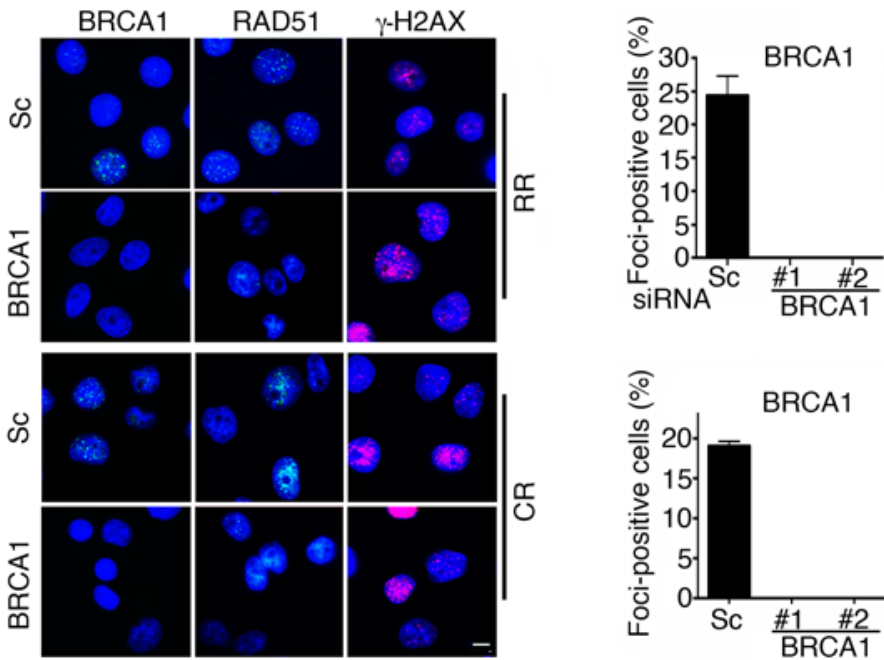

SUM1315MO2-RR1
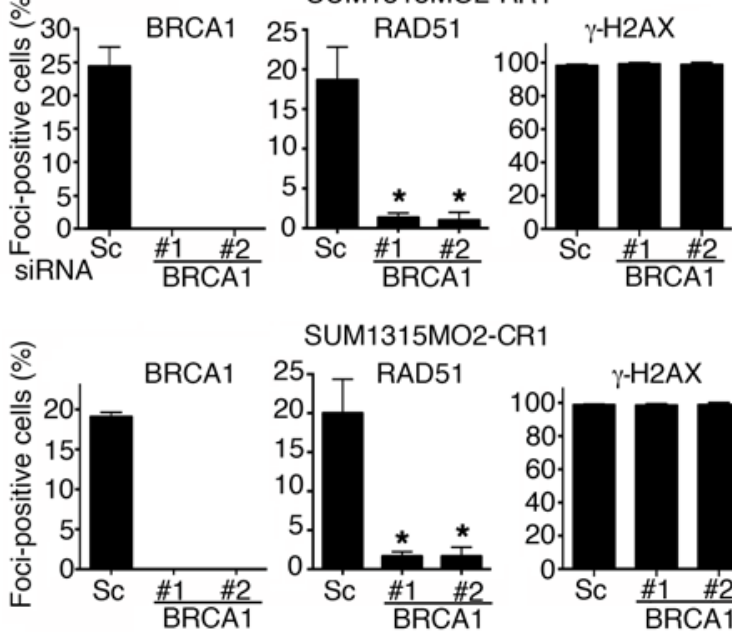

\section{SUM1315MO2-CR1}
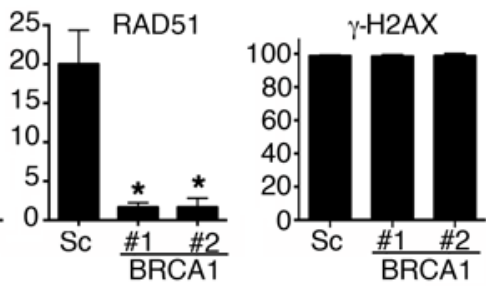

Figure 3. Rdd-BRCA1 does not require BARD1 for stability or function. (A) BRCA1 was immunoprecipitated from MDA-MB-231 and SUM1315MO2 RR1 and CR1 cells using a C-terminal-specific Ab. Immunoprecipitates were measured for BRCA1, BARD1, and CtIP protein levels by Western blot analysis. Three independent experiments were performed. (B) MDA-MB-231, MDA-MB-436-RR1, and SUM1315M02 RR1 cells were treated with scrambled (Sc) or 2 independent siRNAs targeting BRCA1 or BARD1, and BRCA1, BARD1 and tubulin measured by Western blot analysis. Samples were run on parallel gels. The tubulin blot was derived from duplicate samples run on a parallel gel. (C) Cells described in B were transfected with scrambled or 2 independent BARD1 siRNAs, treated with increasing concentrations of rucaparib, and colony formation assessed. (D) Cells were transfected with scrambled, BRCA1 no. 1, or BRCA1 no. 2 siRNA, followed by treatment with increasing concentrations of either rucaparib or cisplatin for either SUM1315MO2 RR1 or CR1 cells, respectively, and then reseeded for colony formation assay $(n=3)$. Insets show Western blots of BRCA1 knockdown. Three independent experiments were performed. (E) SUM1315MO2 RR1 and CR1 cells were treated with scrambled or 2 independent BRCA1 siRNAs and subjected to IR. BRCA1, RAD51, and $\gamma$-H2AX IRIF were assessed by immunofluorescence. Representative images are shown. Scale bar: $10 \mu \mathrm{m}$. Bar graphs show the mean and SEM percentage of cells containing more than 5 foci ( $n=3$ independent experiments; ${ }^{*} P<0.05$, 2-tailed Student's $t$ test). 

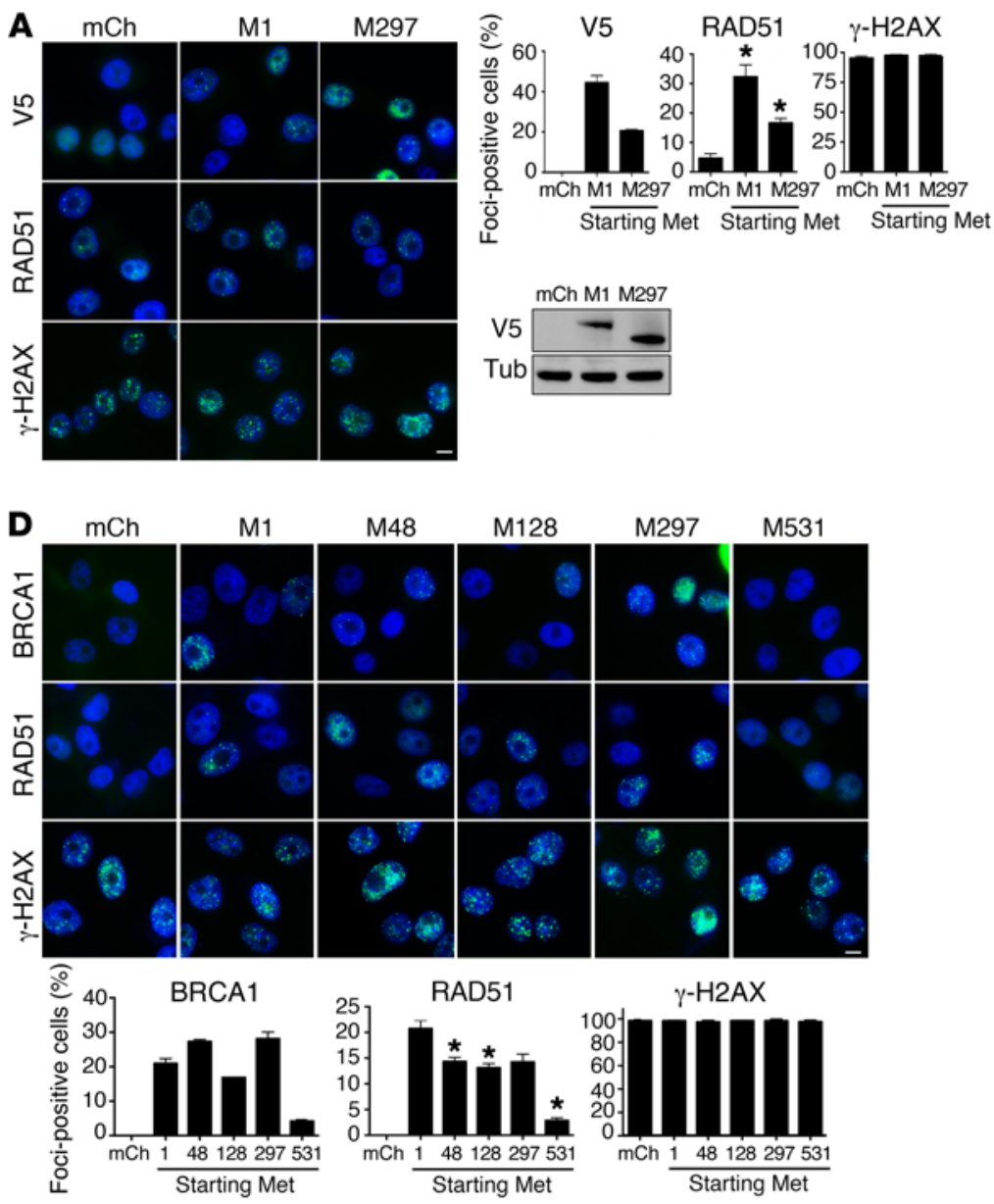

$\gamma-\mathrm{H} 2 \mathrm{AX}$

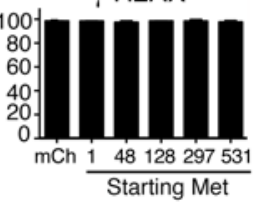

B
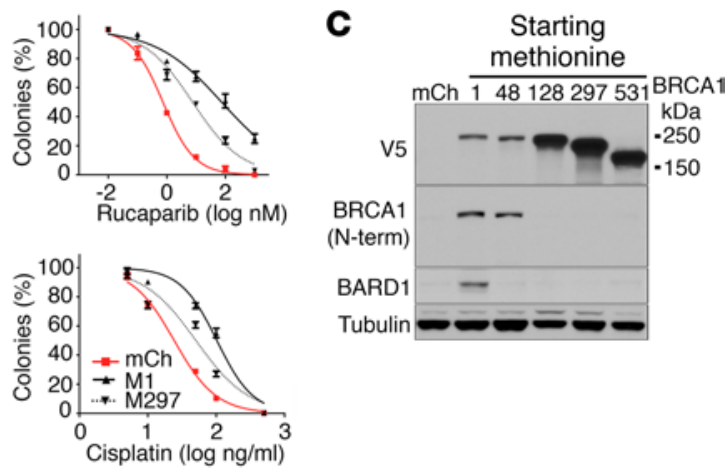

Figure 4. Ectopic overexpression of Rdd-BRCA1 proteins provides partial resistance. (A) SUM1315MO2 parental cells engineered to overexpress mCherry, BRCA1 full-length (M1), and BRCA1-Met-297 (M297) constructs were treated with IR, and V5, RAD51, $\gamma$-H2AX, and DAPI were detected by immunofluorescence. Representative images are shown. Scale bar: $10 \mu \mathrm{m}$. Bar graphs show the mean and SEM percentage of cells containing more than 5 foci $(n=3$ independent experiments; ${ }^{*} P<0.05$, 2-tailed Student's $t$ test). Western blot shows exogenous V5-tagged BRCA1 protein expression. (B) SUM1315M02 cell lines described in A were treated with increasing concentrations of rucaparib or cisplatin and colony formation assessed. Three independent experiments were performed. (C) MDA-MB-436 cells stably expressing mCherry and BRCA1 constructs starting at Met-1 (full-length), Met-48, Met-128, Met-297, and Met-531 were assessed for V5, BRCA1 (N-terminal-specific Ab), BARD1, and tubulin expression by Western blot analysis. (D) MDA-MB-436 cells described in C were treated with IR, and V5, RAD51, $\gamma-\mathrm{H} 2 \mathrm{AX}$, and DAPI were detected by immunofluorescence. Representative images are shown. Scale bar: $10 \mu \mathrm{m}$. Bar graphs show the mean and SEM percentage of cells containing more than 5 foci ( $n=3$ independent experiments; ${ }^{*} P<0.05,2$-tailed Student's $t$ test). (E) MDAMB-436 cells described in C were seeded at 6 different seeding densities and maintained in either $20 \mathrm{nM}$ rucaparib or 20 ng/ml cisplatin for 3 weeks, until resistant colonies emerged or cells died. Representative 6-well plates are shown. Bar graphs show the mean and SEM number of colonies expressed as a percentage of the number of cells originally seeded ( $n=3 ;{ }^{*} P<0.05$, 2-tailed Student's $t$ test). Three independent experiments were performed.

age). However, MDA-MB-231 cells demonstrated robust coverage before Met-297 (79\% coverage), with the first peptide detected at Arg-7. In contrast, we were unable to detect any peptides before Met-297 (0\% coverage), with the first peptide detected at Asn-354 in SUM1315MO2 RR1 cells (Figure 2C and Supplemental Figure 1).

Furthermore, we compared the gel migration of endogenous BRCA1 from SUM1315MO2 RR1 and CR1 cells with exogenously expressed, full-length Met-128- and Met-297-initiating BRCA1 proteins. Gel migration patterns suggested that endogenous BRCA1 from SUM1315MO2 resistant cells migrated in a manner most similar to that of exogenous BRCA1-Met-297 cells (Figure 2D). To confirm that Met-297 was the initiating start site, we used CRISPR/Cas9 gene editing to disrupt the BRCA1 reading frame before (sg_ex2) as well as at the Met-297 coding region (sg_ex11). MDA-MB-231 cells that were BRCA1 WT demonstrated a similar reduction in BRCA1 protein levels with both sg_ex2 and sg_ex11. In contrast, Rdd-BRCA1 protein expression in SUM1315MO2 RR1 cells was not affected by sg_ex2, but decreased with sg_ex11 treatment (Figure 2E and Supplemental Figure 2). These data support the notion that the Rdd -BRCA1 reading frame is initiated at Met-297.

There are several in-frame methionine start sites downstream of the BRCA1 $1^{185 d e l A G}$ mutation; we sought to understand why cells favored Met-297. First, we examined the possibility that alternative mRNA splicing was responsible for removing exons encoding Met-1 (exon 2) and translation start sites before Met-297 (located in exon 11). Reverse transcription-PCR (RT-PCR) analyses using a forward primer located in the $5^{\prime}$-UTR and a reverse primer in exon 11 (downstream of Met-297) detected 1 band that was the predicted size of full-length BRCA1 (Supplemental Figure 3A). Additionally, we carried out a series of quantitative RT-PCR reactions using primers that 

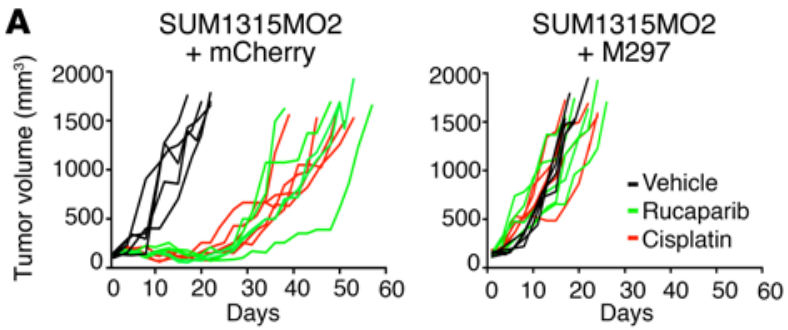

C

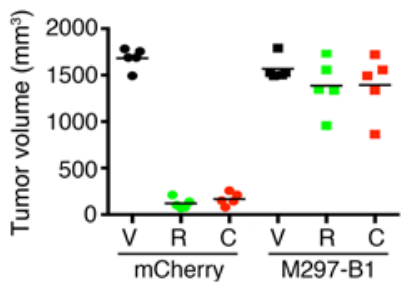

MDA-MB-436

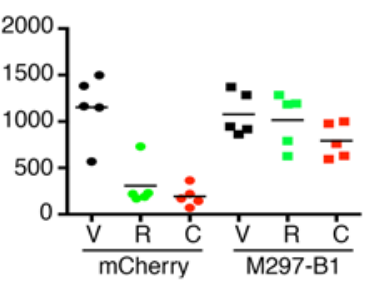

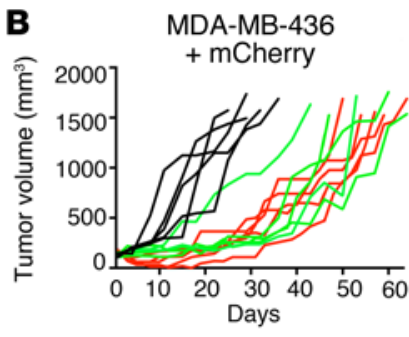

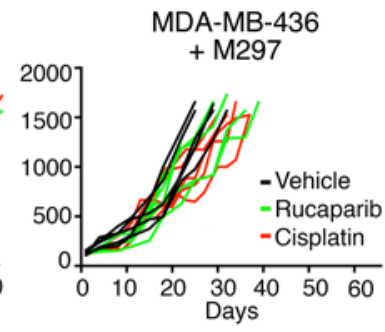

D

SUM1315MO2

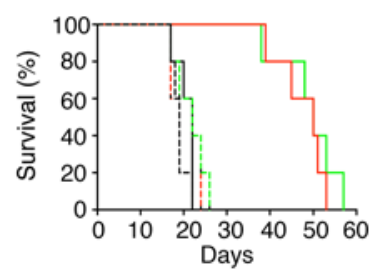

MDA-MB-436

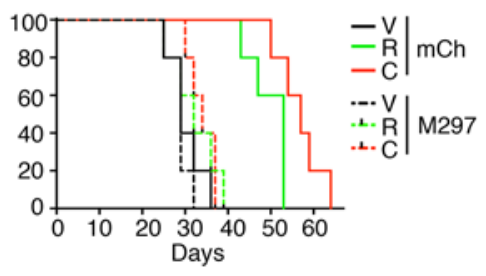

Figure 5. Ectopic overexpression of Rdd-BRCA1 proteins provides resistance in vivo. (A) SUM1315MO2 and (B) MDA-MB-436 tumor xenografts expressing ectopic mCherry or BRCA1-Met-297 were treated with vehicle (black lines), rucaparib (green lines), or cisplatin (red lines), and tumor growth was measured. Each line represents an individual tumor. (C) Individual tumor volumes at day 20 are shown. (D) Kaplan-Meier survival analyses. $n=5$ mice per treatment group.

specifically detected BRCA1 mRNA that contained exons 2, 6, 11, or 16. We found similar relative expression levels for each exon in all cell lines, indicating that full-length $B R C A 1 \mathrm{mRNA}$, rather than an exon-deficient alternative splice isoform, was dominant (Supplemental Figure 3B). We next examined mRNA features that could impact the translation start site choice. Previous studies demonstrated that Met- 48 had a weak, but Met-128 and Met-297 had similar-strength, Kozak consensus motifs (38). Hairpin structures at the 5 ' end of mRNA translation start sites commonly block translation initiation by preventing the $40 \mathrm{~S}$ ribosomal subunit from binding (39-41). Analyses of a BRCA1 mRNA secondary structure revealed a higher than $80 \%$ probability of the presence of a translation initiation inhibitory hairpin structure located immediately before Met-128; however, we found no such structures in proximity to the Met-297 start site (Supplemental Figure 4A) These data reveal the possibility that mRNA sequence and structural features resulted in a preference for Met-297 as the translation initiation site.

DNA damage-induced alternative protein translation is a process in which transcripts utilize downstream translation start sites for protein production, but is accompanied by a global decrease in total cellular translation $(42,43)$. We measured phosphorylated eIF $2 \alpha$ (p-eIF $2 \alpha$ ) and total activating transcription factor 4 (ATF4) protein levels, which were previously shown to be markers of alternative translation (42). SUM1315MO2 RR and CR clones had elevated levels of eIF $2 \alpha$ phosphorylation and ATF 4 compared with levels detected in the parental cell line. Additionally, puromycin incorporation into newly generated peptides was lower in resistant cell lines, suggesting that total levels of global translation were decreased (Supplemental Figure 4, B and C). These data support the notion that the alternative translation pathway contributed to the increased levels of Rdd-BRCA1 protein present in SUM$1315 \mathrm{MO} 2$ resistant derivatives.

Rdd-BRCA1 does not require BARD1 for stability or function. The BRCA1 RING domain mediates interaction with BARD1 and is absent in the BRCA1-Met-297 protein. Both C- and N-termi- nally interacting proteins CtIP and BARD1, respectively, were detected in immunoprecipitates of full-length BRCA1 from MDAMB-231 cells. However, BARD1 was not present in immunoprecipitates of BRCA1 from SUM1315MO2 RR1 or CR1 cells, while CtIP was readily detectable (Figure 3A). Because BARD1 interaction with full-length BRCA1 is required for the stability of both proteins (44), we investigated the impact of siRNAs targeting both BRCA1 and BARD1 on relative protein levels. BRCA1 and BARD1 siRNA treatments resulted in reciprocal depletion of both proteins in MDA-MB-231 and MDA-MB-436 RR1 cells that expressed WT BRCA1 or a C-terminal truncated form of BRCA1, respectively (30). In contrast, BRCA1 siRNA decreased BRCA1 protein levels, but basal BARD1 protein levels were barely detectable and did not change with siRNA treatment in SUM1315MO2 RR1 cells; additionally, BARD1 siRNA did not affect BRCA1 protein levels (Figure 3B). BARD1 siRNA-treated MDA-MB-231 cells were 1,121- $(P<0.001)$ and 945 -fold $(P<0.001)$ more sensitive to rucaparib with 2 independent BARD1 siRNAs than were scrambled siRNA-treated cells. Similarly, MDA-MB-436 RR1 cells were 67- $(P=0.0414)$ and 91 -fold $(P=0.0411)$ more sensitive to rucaparib with 2 independent BARD1 siRNAs than were scrambled siRNA-treated cells. In contrast, BARD1 siRNA did not sensitize SUM1315MO2 RR1 cells to rucaparib treatment (Figure 3C).

To begin to investigate the role of the Rdd-BRCA1 protein in HR DNA repair and drug resistance, we treated RR1 and CR1 cells with either scrambled or 2 independent BRCA1 siRNAs and assessed their impact on rucaparib and cisplatin sensitivity by colony formation assays. BRCA1 siRNA-treated RR1 cells were 11$(P=0.0011)$ and 12 -fold $(P=0.001)$ more sensitive to rucaparib than were scrambled siRNA-treated control cells $(P=0.0002)$. Similarly, CR1 cells treated with BRCA1 siRNAs were $1.9-(P=0.048)$ and 1.7-fold $(P=0.049)$ more sensitive to cisplatin than were scrambled siRNA-treated cells (Figure 3D). Furthermore, BRCA1 siRNA reduced RAD51 foci by $14-(P=0.002)$ and 19 -fold $(P=0.002)$ in RR cells and by $12-(P=0.0019)$ and 12 -fold $(P=0.0021)$ in CR cells, 
A
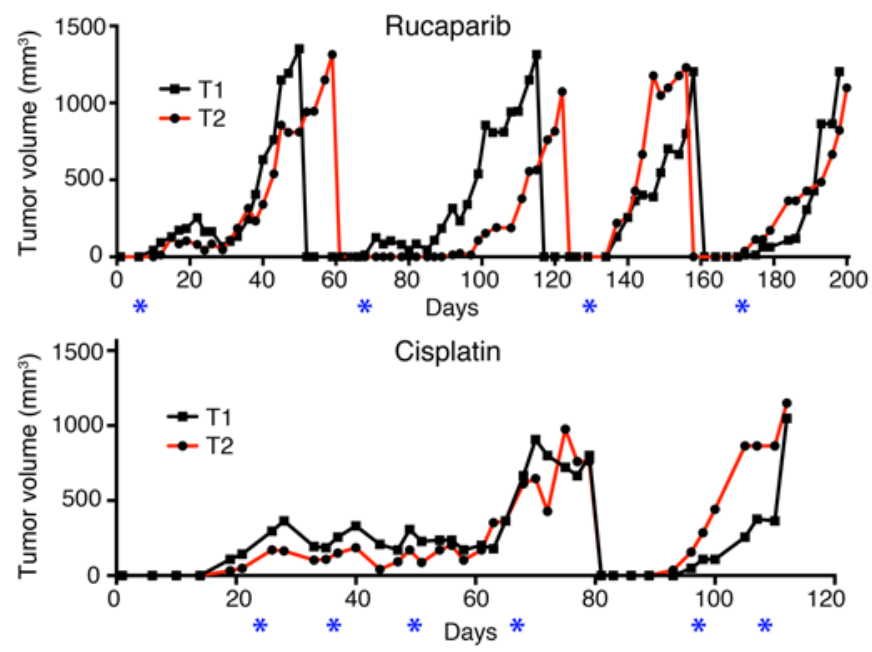

D

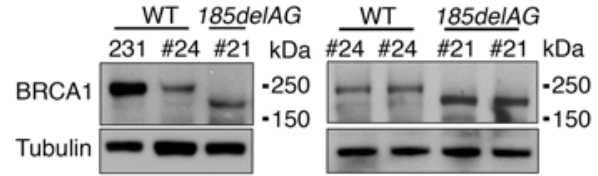

E

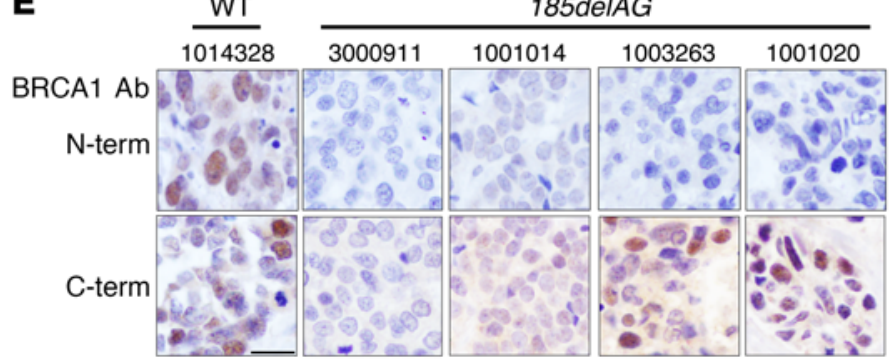

B

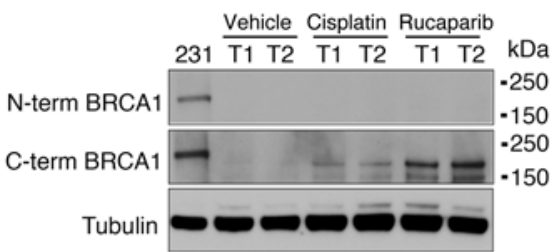

C

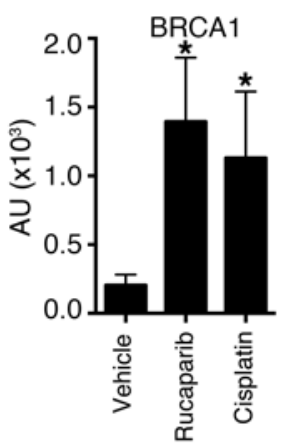

BRCA1 Ab N-term

C-term

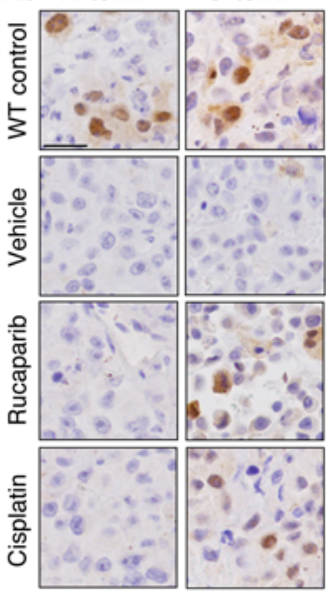

Figure 6. Rdd-BRCA1 proteins are expressed in tumors. (A) Mice carrying SUM1315M02 parental tumors were treated with rucaparib or cisplatin and tumor growth monitored. When tumors reached approximately $1,000 \mathrm{~mm}^{3}$ in size, they were harvested and reimplanted. Red and black lines represent individual tumors from each set of treatments; asterisks represent the days of drug administration. $n=2$ mice per treatment group. (B) Assessment of BRCA1 protein levels from tumors described in A by Western blot analysis. MDA-MB-231 cells were used as BRCA1 WT controls. (C) Bar graph shows quantification of staining intensities using C-terminal Ab of at least 2 individual tumors for each treatment. MDA-MB-231 xenografts were used as BRCA1 WT controls; SUM1315M02 tumors were treated with vehicle or rucaparib, and cisplatin-resistant tumors from $\mathbf{A}$ were assessed by IHC for BRCA1 expression using $\mathrm{N}$ - and C-terminal-specific Abs. Scale bar: $20 \mu \mathrm{m}$. Two tumors for each treatment group were assessed. ${ }^{*} P<0.05$, 2-tailed Student's $t$ test. Bar graph shows quantification of staining intensities using C-terminal Ab of at least 2 individual tumors for each treatment. (D) MDA-MB-231, PDX WO-24 (\#24), and PDX WO-21 (\#21) were assessed by Western blot analysis for BRCA1 protein expression using a C-terminal-specific Ab. Three individual tumors were assessed for each PDX model. Samples were run on parallel gels, and the tubulin blot was derived from duplicate samples run on a parallel gel.

(E) Primary tumors from patients with WT or BRCA1785delAC germline mutations were stained with BRCA1 N-and C-terminal-specific Abs by IHC. Scale bar: $20 \mu \mathrm{m}$. See Supplemental Table 2 for more details.

but $\gamma$-H2AX foci were unaffected (Figure 3E), with little impact on cell-cycle progression (Supplemental Figure 5).

Rdd-BRCA1 proteins are hypomorphic and provide partial resistance in vitro. We next examined the impact of exogenous BRCA1Met-297 overexpression in SUM1315MO2 parental cells. Exogenous full-length and BRCA1-Met-297 both formed IRIF and increased RAD51 IRIF by 6.9- $(P=0.0027)$ and 3.6 -fold $(P=0.0158)$, respectively, compared with mCherry control-expressing cells. The levels of $\gamma$-H2AX were not affected by exogenous protein expression (Figure 4A). Furthermore, SUM1315MO2 cells overexpressing exogenous BRCA1 full-length or Met-297 were 101.3- $(P=0.0255)$ and 10.3-fold ( $P=0.0169)$ more resistant to rucaparib, respectively, as well as 4.3$(P=0.0003)$ and 2.1 -fold $(P=0.0004)$ more resistant to cisplatin than were mCherry-expressing cells, respectively (Figure $4 \mathrm{~B}$ ).
We further assessed the ability of a series of Rdd-BRCA1 proteins to provide PARPi and cisplatin rescue in a cellular background that lacked the endogenous $B R C A 1^{185 d e l A G}$ allele. MDA-MB- 436 cells were selected to assess exogenous Rdd-BRCA1 activity, as these cells harbor a hemizygous $B R C A 1^{5396+1 G>A}$ mutation that disrupts BRCT domain protein folding, resulting in BRCA1 protein degradation and loss of detectable expression; additionally, these cells lack RAD51 foci and are exquisitely PARPi and cisplatin sensitive (30, 35). MDA-MB- 436 cells expressing mCherry control and BRCA 1 proteins initiating at Met-1, $-48,-128,-297$, and -531 were first confirmed by Western blot analysis for exogenous protein expression (Figure 4C). As expected, expression of Met-1, but not the RINGdeficient Met-48-Met-531 proteins, resulted in a reemergence of BARD1. BRCA1-Met-1, -48, -128, and -297, but not Met-531, formed 
efficient BRCA1 IRIF. However, BRCA1-Met-48, -128, -297, and -531 had 1.4- $(P=0.026), 1.6-(P=0.0138), 1.5-(P=0.0671)$, and 7.3-fold $(P=0.0023)$ less RAD51 IRIF compared with that detected in full-length BRCA1-Met-1-expressing cells. The levels of $\gamma$-H2AX were not affected by exogenous protein expression (Figure 4D).

To assess the ability of Rdd-BRCA1 proteins to provide therapy resistance, cells were seeded at a range of densities and incubated with a single rucaparib and cisplatin concentration that selected for drug-resistant colonies. Met-1, -48, -128, and -297 BRCA1-expressing cells had 253- $(P=0.0016), 36-(P<0.001)$, 30- $(P<0.001)$, and 47-fold $(P=0.0033)$ more rucaparib-resistant colonies than did mCherry control cells, respectively; these cells also had 321-, 41-, 55-, and 60-fold (all $P<0.001$ ) more cisplatin-resistant colonies than did mCherry control cells, respectively (Figure 4E). Interestingly, BRCA1-Met-531 had no impact on PARPi or cisplatin rescue. Similar results were obtained using standard colony formation assays exposing cells to increasing concentrations of drug (Supplemental Figure 6, A and B). Additionally, exogenous BRCA1-Met-297 provided rescue in both SUM1315MO2 and MDA-MB-436 cells in the presence of an shRNA targeting only endogenous BRCA1, suggesting that residual endogenous BRCA1 was not contributing to the observed rescue (Supplemental Figure 6, C and D).

BRCA1-Met-297 overexpression promotes resistance in vivo. We assessed the ability of exogenous BRCA1-Met-297 to provide rucaparib and cisplatin resistance in vivo. SUM1315MO2 and MDAMB-436 cells that overexpressed mCherry control or BRCA1-Met297 readily formed tumors and were used for xenograft experiments (Supplemental Figure 7). Rucaparib and cisplatin treatments both inhibited tumor growth in mCherry-expressing SUM1315MO2 and MDA-MB-436 tumors. In contrast, the degree of growth inhibition was markedly reduced in BRCA1-Met-297-expressing tumors (Figure 5, A and B). Twenty days after tumor implantation, rucaparib and cisplatin treatment delayed mean tumor growth by 14$(P<0.0001)$ and 10-fold $(P<0.0001)$ in mCherry-overexpressing SUM315MO2 tumors, respectively, compared with that observed in vehicle-treated mice. Similarly, rucaparib and cisplatin treatment delayed mean tumor growth by 3.7- $(P=0.0023)$ and 5.9 -fold $(P=0.0004)$ in mCherry-overexpressing MDA-MB-436 tumors, respectively, compared with that seen in vehicle-treated mice. In contrast, neither rucaparib nor cisplatin treatment resulted in significant delays in tumor growth in BRCA1-Met-297-overexpressing SUM315MO2 or MDA-MB-436 tumors compared with what was observed in vehicle-treated mice (Figure 5C).

Kaplan-Meier analyses indicated that the median overall survival (OS) of mice harboring SUM1315MO2 mCherry-expressing tumors that were treated with rucaparib or cisplatin increased by 2.3 -fold $(P=0.0034$, log-rank test $)$ and 2.3 -fold $(P=0.0034$, log-rank test), respectively, compared with the median OS of vehicle-treated mice. Similarly, for mice harboring MDA-MB-436 mCherry-expressing tumors that were treated with rucaparib or cisplatin, OS increased by 1.8 -fold ( $P=0.0018$, log-rank test) and 2-fold ( $P=0.0018$, log-rank test), respectively, compared with the OS of vehicle-treated mice. In contrast, mice harboring BRCA1Met-297-expressing tumors that were treated with either rucaparib or cisplatin did not demonstrate significant increases in OS compared with vehicle-treated mice (Figure 5D).
Short-term assessment of tumor response by immunohistochemical analyses demonstrated that rucaparib and cisplatin treatments significantly increased $\gamma-\mathrm{H} 2 \mathrm{AX}$ staining intensity in all tumors. Furthermore, rucaparib and cisplatin treatments decreased Ki67 staining intensity by $3-(P<0.0001)$ and 4.3 -fold $(P<0.0001)$, respectively, in mCherry-expressing SUM1315MO2 tumors and by 2.3 - $(P=0.0046)$ and 2.4 -fold $(P=0.0217)$, respectively, in mCherry-expressing MDA-MB-436 tumors. In contrast, rucaparib and cisplatin treatment did not significantly impact Ki67 staining in BRCA1-Met-297-expressing tumors (Supplemental Figure 8).

$R d d-B R C A 1$ is expressed in resistant tumors. To assess the expression of endogenous Rdd-BRCA1 protein in vivo, SUM$1315 \mathrm{MO} 2$ xenografts that were initially responsive to rucaparib and cisplatin treatment were subjected to multiple rounds of rucaparib or cisplatin treatment until tumors acquired resistance (Figure 6A). Rucaparib- and cisplatin-resistant tumors demonstrated increased expression of Rdd-BRCA1 as measured by Western blot analysis (Figure 6B). Additionally, Abs were optimized to distinguish between $\mathrm{N}$ - and $\mathrm{C}$-terminal-containing BRCA1 proteins by immunohistochemical analyses (Supplemental Figure 7). SUM$1315 \mathrm{MO} 2$ tumors that were rucaparib and cisplatin resistant had a 6.8- $(P<0.0001)$ and 5.5 -fold $(P<0.0001)$ increase in BRCA1 $\mathrm{C}$-terminal $\mathrm{Ab}$ immunohistochemical staining intensity compared with that seen in parental tumors. However, there was no change in N-terminal BRCA1-staining intensity (Figure 6C).

We next compared BRCA1 protein expression levels in a $B R C A 1^{185 d e l A G}$-mutant high-grade serous ovarian cancer (HGSOC) patient-derived xenograft (PDX), WO-21, with levels in a BRCA1 WT HGSOC PDX, WO-24 (Figure 6D). PDX WO-21 was obtained from a patient whose tumor progressed on olaparib therapy and who subsequently underwent tertiary cytoreduction surgery, at which point tumor was collected for orthotopic ovarian transplantation and PDX generation. PDX WO-24 was obtained from an untreated patient during primary cytoreduction surgery. BRCA1 protein expression levels in WO-24 was similar levels in MDAMB-231 cells. However, WO-21, harboring the BRCA1 $1^{185 d e l A G}$ mutation, expressed a truncated BRCA1 protein resembling BRCA1Met-297 (Figure 6D).

Furthermore, we analyzed breast and ovarian carcinomas resected from individual patients with germline $B R C A 1^{185 d e l A G}$ mutations for the expression of Rdd-BRCA1 proteins using $\mathrm{N}$ - and C-terminal-specific Abs (Supplemental Table 2). A patient's carcinoma that was WT for BRCA1 was used as a positive control (specimen 1014328). Here, BRCA1 protein could be detected with both $\mathrm{N}$ - and C-terminal Abs. We did not detect positive BRCA1 staining with either $\mathrm{Ab}$ in 2 primary ovarian carcinomas with $B R C A 1^{185 d e l A G}$ mutations. Interestingly, we were able to detect nuclear BRCA1 staining with the $\mathrm{C}$-terminal, but not the $\mathrm{N}$-terminal, BRCA1 Ab in 2 recurrent breast carcinomas (specimens 1003263 and 1001020) from individuals with $B R C A 1^{185 d e l A G}$ germline mutations (Figure $6 \mathrm{E}$ and Supplemental Table 2).

\section{Discussion}

In the current study, we present evidence to support a mechanism of PARPi and platinum resistance, whereby BRCA1 protein products are generated using translation start sites located downstream of the mutation-induced stop codon. BRCA1 proteins pro- 
duced from downstream translation start sites were truncated at the N-terminal region and lacked the RING domain. However, Rdd-BRCA1 proteins were hypomorphic, contributing to RAD51 loading and PARPi and cisplatin resistance.

Multiple pathogenic frameshifting mutations are located toward the $5^{\prime}$ end of the BRCA1 gene, including the most commonly reported $B R C A 1^{185 d e l A G}$ allele. The $B R C A 1^{185 d e l A G} \mathrm{mRNA}$ is predicted to produce a short, approximately $5-\mathrm{kDa}$ peptide (E23fsX17). Although this peptide has not been detected endogenously, exogenous overexpression of a BRCA1 peptide consisting of aa 1-23fsX17 was reported to induce phenotypes not associated with full-length BRCA1, including enhanced IL-1 $\beta$ expression, and to promote apoptosis $(45,46)$. However, endogenously generated small peptide products normally located in the structured RING region of the protein may not be folded and are likely to be degraded. In line with this, we did not detect any $\mathrm{N}$-terminal region-containing peptide products in SUM1315MO2 parental or resistant cells.

In contrast to the majority of premature termination codoninducing (PTC-inducing) BRCA1 mutations, previous studies demonstrated that $B R C A 1^{185 d e l A G}$ and $B R C A 1^{188 d e l 11}$ alleles were not subject to nonsense-mediated mRNA decay (NMD), leading to speculation that translation may continue after the PTC and prevent NMD $(38,47)$. Using C-terminal-specific BRCA1 Abs, we confirmed that translation does occur after the $B R C A 1^{185 d e l A G_{2}}$ induced PTC, and we readily identified a protein product that was only slightly smaller in size compared with that of fulllength BRCA1 in SUM1315MO2 parental and resistant cells, probably starting at Met-297. Previous in silico analyses predicted Met-128 and Met-297 to be downstream BRCA1 translation start sites (38). Our analyses suggest that BRCA1 mRNA structural features may result in a preference for the Met-297 translation initiation site.

BRCA1-Met-297 was present in parental SUM1315MO2 cells but more abundant in rucaparib and cisplatin resistant clones. Interestingly, expression of BRCA1-Met-297 tended to be higher in cells in which resistance was rucaparib induced as opposed to cisplatin induced. This could be reflective of the type of DNA repair mechanisms that are induced by each agent, whereby PARPi primarily activates BRCA1 mediated-HR DNA repair, whereas platinum is likely to elicit both HR and nucleotide excision repair (NER) DNA repair pathways (48). Alternatively, PARPi may have a greater impact on DNA damage-induced alternative translation pathways (43). DNA damage has previously been associated with translational reprogramming, allowing for selective synthesis of DNA damage response proteins, and relies on upstream ORFs; DNA damage has also been associated with a general reduction in global translation $(42,43,49)$. Our data suggested that SUM$1315 \mathrm{MO} 2$ resistant cells had lower rates of global translation as well as increased levels of p-eIF $2 \alpha$ and total ATF4, potentially indicating that the alternative translation pathway contributed to increased BRCA1-Met-297 expression.

In contrast to full-length BRCA1, Rdd-BRCA1 did not require BARD1 for stability, probably due to an absence of the RINGlocated nuclear export signal usually masked by BARD1 binding (50). However, BARD1 was unstable and present at low or undetectable levels in the absence of a BRCA1 RING domaincontaining peptide. Previous studies using genetic mouse mod- els of mammary carcinogenesis demonstrated that the Brca1 ${ }^{\mathrm{C} 61 G}$ allele product, which is unable to interact with BARD1, promoted RAD51 loading as well as platinum and PARPi resistance (31). The mechanism we describe may not be limited to cancers with $B R C A 1^{185 d e l A G}$ mutations and could be relevant to multiple frameshifting, 5'-located BRCA1 mutations. However, mutations located after Met-297 (c.891) are unlikely to develop resistance through this mechanism, as the next downstream translation start site at Met-531 (c.1593) produced a functionless protein. Truncated constructs expressing BRCA1 BRCT domains have previously been shown to be capable of forming foci (51-55). However, BRCA1-Met-531 did not form IRIF, despite retaining BRCT domains, suggesting that the peptide length or specific regions retained may impact the ability of BRCT domains to be recruited to sites of DNA damage.

SUM1315MO2 cells and tumors were initially PARPi and platinum sensitive, in line with the patients' clinical response data, in which many patients harboring $B R C A 1^{185 d e l A G}$ mutations had robust PARPi or platinum responses, but eventually acquired resistance $(17,56)$. Although RNAi-mediated depletion of endogenous Rdd-BRCA1 from SUM1315MO2 RR and CR cells reduced RAD51 IRIF and restored PARPi and cisplatin sensitivity, ectopic overexpression of BRCA1-Met-297 did not provide the same degree of resistance that was observed in SUM1315MO2 RR and CR cells. Furthermore, RING domain-deficient BRCA1 proteins were less effective at rescuing PARPi and cisplatin sensitivity than were ectopic full-length BRCA1-expressing cells. These data suggest that Rdd-BRCA1 proteins were important for the development of resistance; however, additional epi/genetic events may work in conjunction with BRCA1-Met-297 to generate a more robust resistance phenotype.

$B R C A 1^{185 d e l A G}$ tumors from patients who had developed either platinum or PARPi resistance were previously shown to frequently harbor genetic reversion mutations that restored the WT sequence (22). Recent analyses of therapy-resistant ovarian carcinomas confirmed that approximately half of BRCA1-mutant carcinomas tested harbored secondary mutations that restored the ORF (25). Interestingly, the allele frequency of reversion mutations often varied within a tumor, potentially suggesting that intratumoral heterogeneity facilitates the existence of more than one mechanism of resistance within individual carcinomas. It is possible that reversion mutation-containing cells coexist with RING-deficient BRCA1 protein-expressing cells within neoplasms.

In conclusion, we show that BRCA1 RING domain-deficient proteins retain hypomorphic activity and, when expressed at high enough levels, can contribute to HR DNA repair and therapy resistance. In light of the increasing number of resistance mechanisms described to date, multiple biological events may contribute to therapy resistance in $B R C A 1$ mutation carriers, as well as within individual tumors. Further work is required to assess the incidence of these events in patients' malignancies as well as their impact on chemotherapeutic response and survival outcomes.

\section{Methods}

Cell lines and reagents. Cell lines were obtained from the ATCC or Asterand. Cell lines tested negative for mycoplasma. Cells were authenticated by DNA fingerprinting (IDEXX BioResearch). Chem- 
icals were purchased from Sigma-Aldrich. Clovis Oncology provided rucaparib (CO-338). Cisplatin was from APP/Fresenius Kabai USA and placlitaxel from Sagent Pharmaceuticals.

Derivation of resistant clones. SUM1315MO2 cells were cultured in the presence of either $1 \mu \mathrm{M}$ rucaparib or $30 \mathrm{ng} / \mathrm{ml}$ cisplatin in 6-well plates until 3 independent resistant clones emerged (approximately 1 month). Clones were labeled RR1 to RR3 and CR1 to CR3. Cells were cultured in the absence of rucaparib or cisplatin for a minimum of 2 weeks before they were used for experiments.

Colony formation assays. Cells were seeded in 6-well plates at 1,000 cells per well in the presence of increasing concentrations of rucaparib. For cisplatin, mitomycin C, or taxol treatments, exponentially growing cells were cultured in 24-well plates, treated with increasing concentrations or doses for 24 hours, and replated at 1,000 cells per well in 6 -well plates for colony formation. For siRNA treatments, exponentially growing cells were reverse transfected in 24-well plates, and 2 days after transfection, cells were treated with rucaparib for 72 hours or cisplatin for 24 hours and then replated in 6-well plates for colony formation. For the resistance emergence assays described in Figure 4E, cells were seeded at decreasing densities in 6-well plates and maintained in the presence of either $20 \mathrm{nM}$ rucaparib or $20 \mathrm{ng} / \mathrm{ml}$ cisplatin until resistant colonies emerged. Colony formation was assessed 2 weeks after plating with crystal violet staining. The mean colony formation from 3 experiments was expressed as a percentage of colonies \pm SEM relative to vehicle-treated cells. Lethal concentration $\left(\mathrm{LC}_{50}\right)$ values (concentration required to reduce colony formation by $50 \%$ compared with vehicle) were calculated using GraphPad Prism software (Version 6) and used to compare fold changes in drug sensitivity.

Immunoprecipitation, mass spectrometry, Western blotting, immunofluorescence, and focal microscopy. BRCA1 Ab (Santa Cruz Biotechnology Inc.; catalog sc-6954) was used for immunoprecipitation of BRCA1 complexes from $2 \mathrm{mg}$ nuclear extract using a Pierce Classic IP Kit (Thermo Fisher Scientific) according to the manufacturer's instructions. Nuclear extracts were derived using NE-PER Nuclear and Cytoplasmic Extraction Reagents (Thermo Fisher Scientific) according to the manufacturer's instructions. For peptide analyses, after immunoprecipitation, bands corresponding to BRCA1 were cut out and gel purified, digested using chemotrypsin, and analyzed by liquid chromatography-tandem mass spectrometry (LC-MS/MS). The following Abs were used to detect proteins by Western blotting: tubulin (Cell Signaling Technology; catalog 2148); HSP90 (Enzo Life Sciences; catalog ADI-SPA-830); RAD51 (Santa Cruz Biotechnology Inc., catalog sc-8349); N-terminal BRCA1, MS110 (Calbiochem; catalog OP92); C-terminal BRCA1 (Santa Cruz Biotechnology Inc.; catalog sc-6954); C-terminal BRCA1 (EMD Millipore; catalog 07-434); V5 (Bethyl Laboratories; catalog A190-120A); CtIP (Bethyl Laboratories; catalog A300-488A); BARD1 (Bethyl Laboratories; catalog A300-263A); p-S51 eIF2 $\alpha$ (Cell Signaling Technology; catalog 3398); total eIF2 $\alpha$ (Cell Signaling Technology; catalog 9722); and ATF4 (Cell Signaling Technology; catalog 11815). For assessment of newly translated peptides, cells were incubated with $1 \mu \mathrm{M}$ puromycin for 30 minutes. Cells were then collected and subjected to Western blot analysis using anti-puromycin Ab (Kerafast; catalog EQ0001). For immunofluorescence, V5 (Bethyl Laboratories; catalog A190-120A); BRCA1 (Santa Cruz Biotechnology Inc.; catalog sc-6954); $\gamma$-H2AX [pS139] (EMD Millipore; catalog 05-636); and RAD51 (Santa Cruz Biotechnology Inc.; catalog sc-8349), Abs were followed by second- ary Abs conjugated to FITC or Texas Red (Jackson ImmunoResearch Laboratories; codes 711-585-152, 711-095-152, 715-095-150, and 715585-150). We acquired immunofluorescence images using a Nikon NIU Upright Fluorescence Microscope and generated images using Nikon NIS Elements software. For IR experiments, we routinely fixed cells 8 hours after treatment with 10 Gy. For analyses, we counted a minimum of 200 cells per condition or cell line. Each experiment was performed at least 3 times with biological replicates.

Cell-cycle analysis. Cells were harvested and fixed with ethanol. The cells were washed with PBS and resuspended in $0.5 \mathrm{ml} \mathrm{FxCycle}$ PI/RNase Staining Solution (Life Technologies, Thermo Fisher Scientific) and incubated at room temperature for 30 minutes. The data were acquired using a BD LSR II Flow Cytometer and analyzed using FlowJo software.

CRISPR/Cas9 gene editing. The CRISPR/Cas9 system was a gift of Christoph Seeger (Fox Chase Cancer Center) (57). MDA-MB-231 and SUM1315MO2 cells were first infected with the lentiviral vector pCW-Cas 9 and selected with puromycin $(2 \mu \mathrm{g} / \mathrm{ml})$. Cas9 expression was conditionally induced by the presence of doxycycline $(4 \mu \mathrm{g} / \mathrm{ml})$. A Cas9 high-expression clone was selected for sequential genome editing. Gene-specific single-guide RNA (sgRNA) sequences were identified using the CRISPR Design Tool (http://crispr.mit.edu/). We used the following DNA oligonucleotides to generate the following sgRNAs: sg_GFP, forward: CACCGAGCTGGACGGCGACGTAAA; sg_GFP, reverse: AAACTTTACGTCGCCGTCCAGCTC; sg_exon 2, forward: CACCGTTGTGCTGACTTACCAGAT; sg_exon 2, reverse: AAACATCTGGTAAGTCAGCACAAC; sg_exon 11, forward: CACCGTGAAGTTAACAAATGCACC; sg_exon 11, reverse: AAACGGTGCATTTGTTAACTTCAC. sgRNA oligonucleotides were cloned into the sgRNA expression vector PLX-SG1 using BsmBI sites.

MDA-MB-231-Cas9 and SUM1315MO2-Cas9 cells were then infected with the lentiviral vector PLX-SG1 and selected with blasticidin $(10 \mu \mathrm{g} / \mathrm{ml})$. After a 5-day incubation with doxycycline $(4 \mu \mathrm{g} / \mathrm{ml})$, cells were collected. For validation of targeted mutations, genomic DNA was isolated from cells, followed by PCR amplification of targeted loci using the forward and reverse primers listed below, and PCR products were directly sequenced using the primers listed below. The expression of BRCA1 was further confirmed by Western blot analysis. Exon 2, forward: GATAGGAACTGGAATATGCCTTGA, exon 2, reverse: CAGCCTCTCGACAGAGATCCTAT, exon 2, sequenced: ATTGGAGAAAGCTAAGGCTACCA; exon 11, forward: GAGTGGTTTTCCAGAAGTGATGA, exon 11, reverse: TAATACTGGAGCCCACTTCATTAGT, exon 11, sequenced: TGGCTCAGTAACAAATGCTCCTAT.

RNA interference and CDNA add-back treatments. We purchased Hs_BRCA1_FlexiTube siRNA constructs (construct 1, SI00096313 and construct 2, SI02654575) and AllStars Negative Control siRNA (scrambled control) from QIAGEN. BARD1 siRNA 1 (J-003873-09) and siRNA 2 ( J-003873-10) were obtained from Dharmacon. Mission shRNA nontarget control (SHCOO2) was purchased from Sigma-Aldrich, and shRNA targeting endogenous BRCA1 was a gift of Jos Jonkers (Netherlands Cancer Institute, Amsterdam, Netherlands). Transfections were carried out according to standard protocols. mCherry and HA-BRCA1 cDNA were cloned into the pENTR1A Gateway Entry vector (Invitrogen) and shuttled into a pDest-IRES-GFP Destination vector (Life Technologies, Thermo Fisher Scientific). To generate BRCA1 proteins where translation initiated at methionines $48-531$, a small fragment corresponding to the specific methionine of interest was first amplified using the follow- 
ing primers: Met-48, forward: CCCAAGCTTATGCTGAAACTTCTCAACCAGAAG, reverse: ATCTGTTATGTTGGCTCCTTGCTA; Met128, forward: CCCAAGCTTATGGGCTACAGAAACCGTGC, reverse: ATCTGTTATGTTGGCTCCTTGCTA; Met-297, forward: AGCTTATGAATGTAGAAAAGGCTG, reverse: AATTCAGCCTTTTCTACATTCATA; Met-531, forward: CCCAAGCTTATGATAAATCAGGGAACTAACCAAAC, reverse: CGAGATACTTTCCTGAGTGCCA. The amplified fragments were used to replace the region spanning the HA affinity tag to the ATG of interest in a pENTR-HA-BRCA1-V5 (full-length) construct using HindIII and EcoRI (M48, M128, M297) and KpnI (M531) sites. cDNAs were shuttled into pLenti-IRES-GFP Destination vectors using the LR Clonase system (Invitrogen). Lentiviral generation and infections were carried out according to standard protocols. Protein knockdown or reexpression was routinely assessed 72 hours after transfection or 96 hours after infection. Cells infected with cDNAs were sorted for GFP positivity using the BD FACSAria II cell sorter and routinely checked for GFP positivity to maintain stable cell lines.

Gene-sequencing and RNA analyses. Genomic DNA was isolated from cells using the DNeasy Blood and Tissue kit (QIAGEN). BRCA1 gene sequencing was carried out as previously described (58). PCR amplicons were sequenced bidirectionally using the Applied Biosystems Big Dye Terminator version 3.1 Cycle Sequencing Kit and an ABI 3130xl genetic analyzer. Trace sequences were analyzed using Sequencher version 4.9 software (Gene Codes Corporation) and ABI Sequence Scanner version 1.0 software. BROCA sequencing included the following genes: ATM, ATR, BABAM1, BAP1, BARD1, BLM, BRCA1, BRCA2, BRCC36, BRE, BRIP1, CDK12, CHEK1, CHEK2, DCLRE1C, FAM175A, FANCC, ID4, LIG4, MLH1, MRE11A, MSH2, MSH6, NBN, PALB2, PIK3CA, PMS2, PRKDC, PTEN, RAD5O, RAD51, RAD51B, RAD51C, RAD51D, RBBP8, SLX4, TOPBP1, TP53, TP53BP1, UIMC1, USP28, XRCC2, XRCC3, XRCC4, XRCC5, and XRCC6, and only clear loss-of-function mutations were reported (37).

Total RNA was isolated from cell lines using the RNeasy Plus Mini Kit (QIAGEN). RT-PCR assays were performed with cDNA generated using the SuperScript III First-Strand Synthesis System (Life Technologies, Thermo Fisher Scientific) and Hot Start Taq DNA Polymerase (New England BioLabs). Primers were located in exon 1, forward: GTATTCTGAGAGGCTGCTGCTTAG and exon 11, reverse: TTCATTTGGCTTGTTACTCTTCTTG.

For quantitative RT-PCR, RNA was tested for quality on a Bioanalyzer (Agilent). RNA concentrations were determined with a spectrophotometer (NanoDrop; Thermo Fisher Scientific). RNA was reverse transcribed using Moloney murine leukemia virus reverse transcriptase (Ambion, Thermo Fisher Scientific) and a mixture of anchored oligo-dT and random decamers. Two reverse transcription reactions were performed for each sample using either 100 or $25 \mathrm{ng}$ input RNA. Assays were used in combination with Taqman Universal Master Mix or Power SYBR Green Master Mix and run on a 7900 HT Sequence Detection System (all from Applied Biosystems, Thermo Fisher Scientific). Cycling conditions were $95^{\circ} \mathrm{C}$ for 15 minutes, followed by 40 (2-step) cycles $\left(95^{\circ} \mathrm{C}, 15 \mathrm{~s} ; 60^{\circ} \mathrm{C}, 60 \mathrm{~s}\right)$. Ct values were converted to quantities (in $\mathrm{AU}$ ) using a standard curve (4 points, 4-fold dilutions) established with a calibrator sample. BRCA1 and the housekeeping gene POLR2F mRNA levels were measured using the following forward and reverse primers: BRCA1, exons $2 / 3$, forward: TTATCTGCTCTTCGCGTTGAAG, BRCA1, exons 2/3, reverse: TTGTGGAGACAGGTTCCTTGAT; BRCA1, exons 6/7, forward:
TGACACAGGTTTGGAGTATGCA, BRCA1, exons 6/7, reverse: CTGTAGCCCATACTTTGGATGATAGA; BRCA1, exon 11, forward: TAGCAAGGAGCCAACATAACAGAT, BRCA1, exon 11, reverse: CTGTAGCCCATACTTTGGATGATAGA; BRCA1, exons 15/16, forward: GAATAGAAACTACCCATCTCAAGAGGA, BRCA1, exons 15/16, reverse: CAGGTAAGGGGTTCCCTCTAGAT; POLR2F, forward: TGCCATGAAGGAACTCAAGG, POLR2F, reverse: TCATAGCTCCCATCTGGCAG.

For each experiment, the BRCA1 mRNA values were normalized to $P O L 2 R F$ values and expressed as a fraction of expression levels detected in MDA-MB-231 cells.

RNA secondary structure predicted was made using the RNAstructure web server (http://rna.urmc.rochester.edu/RNAstructureWeb/Servers/Predict1/Predict1.html). Briefly, we entered the 70-bp mRNA sequence upstream of Met-128 and Met-297 for secondary mRNA prediction using the default settings.

PDX tumor derivation, xenograft treatments, and analyses. NSG

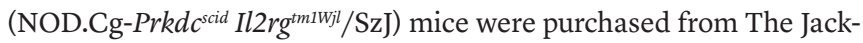
son Laboratory. Patients' tumors were harvested at the time of surgery, and small tumor chunks (5-mm) were transplanted into the ovary of 5- to 8-week-old female mice. Once tumors were established and reached approximately 700 to $1,000 \mathrm{~mm}^{3}$ by ultrasound (M-Turbo; SonoSite), tumors were harvested for expansion and frozen for DNA, RNA, and protein analyses. Whole-exome sequencing was performed for BRCA1 and BRCA2 genes.

For xenograft studies, SUM1315MO2 and MDA-MB-436 cells were s.c. implanted into 6-week-old female NSG mice. When tumors reached approximately $300 \mathrm{~mm}^{3}$, they were harvested and cut into smaller pieces, followed by s.c. reimplantation. Treatment was initiated when tumors reached between 150 and $180 \mathrm{~mm}^{3}$ in size. Rucaparib was administered at $150 \mathrm{mg} / \mathrm{kg}$ twice daily for 10 consecutive days, with a 2-day break after the first 5 days. Cisplatin was administered in a single dose of $6 \mathrm{mg} / \mathrm{kg}$. Vehicle treatment consisted of $0.5 \%$ methylcellulose in water. Tumors were measured with calipers and tumor volumes calculated using the formula: (length $\times$ width ${ }^{2}$ ). Measurements were made every 3 days and mice euthanized when tumors reached $1,500 \mathrm{~mm}^{3}$, in accordance with the institutional guidelines of Fox Chase Cancer Center.

Histologic and immunohistochemical staining. Slides were deparaffinized and hydrated. Antigen retrieval was performed using EDTA buffer, pH 9 (Dako; code S2368). Endogenous peroxidases were quenched by the immersion of slides in $3 \%$ hydrogen peroxide solution (30\% $\mathrm{H}_{2} \mathrm{O}_{2}$, Fisher BP2633-500, diluted in methanol). The following primary Abs were used: Ki-67 (Epitomics; clone EP5; 1:1,500) or $\gamma$-H2AX (pS139) (EMD Millipore; N1-431;1:20,000); BRCA1 N-terminal (Calbiochem; MS110; 1:400); and BRCA1 C-terminal (EMD Millipore; 07-434; 1:7,500). Abs were diluted with DaVinci Green Diluant (Biocare Medical; code PD900) and incubated on slides overnight at $4^{\circ} \mathrm{C}$ in a humidified slide chamber. Slides were then washed 3 times in Tris-buffered saline with Tween-20 (TBST) and incubated with EnVision+System HRP Labelled Polymer Anti-Rabbit (Dako; code K4003) for 1 hour at room temperature. Specimens were washed 3 times in TBST and then developed with DAB solution (Dako; code K3468) and counterstained in Meyer's Hematoxylin (Sigma-Aldrich; catalog MHS32-1L). For analyses of Ki67 and $\gamma$-H2AX expression, a minimum of 2 tumors derived from 2 separate mice were used. Mice were treated with rucaparib $150 \mathrm{mg} / \mathrm{kg} 2$ times per day for 4 consecutive 
days. For cisplatin, mice were treated with a single dose of $6 \mathrm{mg} / \mathrm{kg}$. Tumors were resected and formalin fixed 4 days after the first dose. A minimum of 2 tumors and 3 images per tumor were used to calculate staining intensities. Image-staining intensities were measured using Image J software (NIH). For BRCA1 patient tumor analyses, staining was scored either positively or negatively, on the basis of the presence or absence of nuclear staining.

Statistics. All results are presented as the mean \pm SEM and were analyzed by a 2-tailed Student's $t$ test. Statistical significance was accepted at a $P$ value of less than 0.05 .

Study approval. Fox Chase Cancer Center (IRB no. 14-805) and the University of Pennsylvania (IRB no. 702679) approved the work carried out in this study. Animals were maintained according to IACUC guidelines of the Fox Chase Cancer Center (IACUC no. 12-13) and the Wistar Institute (Philadelphia, Pennsylvania, USA; IACUC no. 112635).

\section{Author contributions}

YW, AJB, JJK, EN, KQC, HHK, EG, FS, and NJ designed and performed the experiments. MIH and EMS carried out BROCA analyses. YW, AJB, JJK, EN, HHK, EG, FS, and NJ analyzed and interpreted the data. NJ wrote the manuscript and supervised the project.

\section{Acknowledgments}

This work was supported by the National Cancer Institute (NCI) (5P30CA006927, to the Fox Chase Cancer Center); the Fox Chase Cancer Center-University of Pennsylvania (FCCC-UPENN) Specialized Program of Research Excellence (SPORE) in Ovarian Cancer; a Susan G. Komen Career Catalyst Award (CCR12226280, to NJ); a Department of Defense Pilot Award and Nested Teal Postdoctoral Scholar Award (OC140040, to NJ and YW) and OC130212 Department of Defense Ovarian Academy Award (NJ); an NIH T32 Postdoctoral Trainee Grant (T32 CA 9035-39, to JJK); the Pacific Ovarian Cancer Research Consortium SPORE in Ovarian Cancer (P50CA83636) and the Wendy Feuer and OCRF Program Project Development Grant (both to EMS). Clovis Oncology supplied rucaparib. We are grateful to Cara Dubyk for help with IHC staining and to the Fox Chase Cancer Center Biorepository, Cell Sorting, Genomics, and Histopathology facilities. We thank Hsin Yao Tang at the Wistar Proteomics facility for help with mass spectrometry.

Address correspondence to: Neil Johnson, Fox Chase Cancer Center, 333 Cottman Avenue, Philadelphia, Pennsylvania 19111, USA. Phone: 215.728.7016; Email: neil.johnson@fccc.edu.
1. Szabo CI, King MC. Inherited breast and ovarian cancer. Hum Mol Genet. 1995;4(spec no):1811-1817.

2. Friedman LS, et al. Confirmation of BRCA1 by analysis of germline mutations linked to breast and ovarian cancer in ten families. Nat Genet. 1994;8(4):399-404.

3. Yang X, Lippman ME. BRCA1 and BRCA2 in breast cancer. Breast Cancer Res Treat. 1999;54(1):1-10.

4. Petrucelli N, Daly MB, Feldman GL. Hereditary breast and ovarian cancer due to mutations in BRCA1 and BRCA2. Genet Med. 2010;12(5):245-259.

5. Roy R, Chun J, Powell SN. BRCA1 and BRCA2: different roles in a common pathway of genome protection. Nat Rev Cancer. 2012;12(1):68-78.

6. Huen MS, Sy SM, Chen J. BRCA1 and its toolbox for the maintenance of genome integrity. Nat Rev Mol Cell Biol. 2010;11(2):138-148.

7. Jiang Q, Greenberg RA. Deciphering the BRCA1 Tumor Suppressor Network. J Biol Chem. 2015;290(29):17724-17732.

8. Moynahan ME, Chiu JW, Koller BH, Jasin M. Brca1 controls homology-directed DNA repair. Mol Cell.1999;4(4):511-518.

9. Bryant HE, et al. Specific killing of BRCA2-deficient tumours with inhibitors of poly(ADP-ribose) polymerase. Nature. 2005;434(7035):913-917.

10. Farmer H, et al. Targeting the DNA repair defect in BRCA mutant cells as a therapeutic strategy. Nature. 2005;434(7035):917-921.

11. Moynahan ME, Cui TY, Jasin M. Homologydirected dna repair, mitomycin-c resistance, and chromosome stability is restored with correction of a Brca1 mutation. Cancer Res. 2001;61(12):4842-4850.

12. Quinn JE, et al. BRCA1 functions as a differential modulator of chemotherapy-induced apoptosis. Cancer Res. 2003;63(19):6221-6228.

13. Silver DP, et al. Efficacy of neoadjuvant Cisplatin in triple-negative breast cancer. J Clin Oncol. 2010;28(7):1145-1153.

14. Safra T, Rogowski O, Muggia FM. The effect of germ-line BRCA mutations on response to chemotherapy and outcome of recurrent ovarian cancer. Int J Gynecol Cancer. 2014;24(3):488-495.

15. Jayson GC, Kohn EC, Kitchener HC, Ledermann JA. Ovarian cancer. Lancet. 2014;384(9951):1376-1388.

16. Scott CL, Swisher EM, Kaufmann SH. Poly (ADP-ribose) polymerase inhibitors: recent advances and future development. J Clin Oncol. 2015;33(12):1397-1406.

17. Kaufman B, et al. Olaparib monotherapy in patients with advanced cancer and a germline BRCA1/2 mutation. JClin Oncol. 2015;33(3):244-250.

18. Ledermann J, et al. Olaparib maintenance therapy in patients with platinum-sensitive relapsed serous ovarian cancer: a preplanned retrospective analysis of outcomes by BRCA status in a randomised phase 2 trial. Lancet Oncol. 2014;15(8):852-861.

19. Luvero D, Milani A, Ledermann JA. Treatment options in recurrent ovarian cancer: latest evidence and clinical potential. Ther Adv Med Oncol. 2014;6(5):229-239.

20. Ledermann JA, Kristeleit RS. Optimal treatment for relapsing ovarian cancer. Ann Oncol. 2010;21(suppl 7):vii218-vii222.

21. Lord CJ, Ashworth A. Mechanisms of resistance to therapies targeting BRCA-mutant cancers. Nat Med. 2013;19(11):1381-1388.

22. Norquist B, et al. Secondary somatic mutations restoring BRCA1/2 predict chemotherapy resistance in hereditary ovarian carcinomas. J Clin Oncol. 2011;29(22):3008-3015.

23. Sakai W, et al. Secondary mutations as a mechanism of cisplatin resistance in BRCA2-mutated cancers. Nature. 2008;451(7182):1116-1120.

24. Edwards SL, et al. Resistance to therapy caused by intragenic deletion in BRCA2. Nature.
2008;451(7182):1111-1115.

25. Patch AM, et al. Whole-genome characterization of chemoresistant ovarian cancer. Nature. 2015;521(7553):489-494.

26. Rottenberg S, et al. High sensitivity of BRCA1-deficient mammary tumors to the PARP inhibitor AZD2281 alone and in combination with platinum drugs. Proc Natl Acad Sci US A. 2008;105(44):17079-17084.

27. Bunting SF, et al. 53BP1 inhibits homologous recombination in Brca1-deficient cells by blocking resection of DNA breaks. Cell. 2010;141(2):243-254.

28. Bouwman P, et al. 53BP1 loss rescues BRCA1 deficiency and is associated with triple-negative and BRCA-mutated breast cancers. Nat Struct Mol Biol. 2010;17(6):688-695.

29. Jaspers JE, et al. Loss of 53BP1 causes PARP inhibitor resistance in Brca1-mutated mouse mammary tumors. Cancer Discov. 2013;3(1):68-81.

30. Johnson N, et al. Stabilization of mutant BRCA1 protein confers PARP inhibitor and platinum resistance. Proc Natl Acad Sci US A. 2013;110(42):17041-17046.

31. Drost R, et al. BRCA1 RING function is essential for tumor suppression but dispensable for therapy resistance. Cancer Cell. 2011;20(6):797-809.

32. Shakya R, et al. BRCA1 tumor suppression depends on BRCT phosphoprotein binding, but not its E3 ligase activity. Science. 2011;334(6055):525-528.

33. Friedman LS, et al. Novel inherited mutations and variable expressivity of BRCA1 alleles, including the founder mutation 185delAG in Ashkenazi Jewish families. Am J Hum Genet. 1995;57(6):1284-1297.

34. Struewing JP, et al. The carrier frequency of the BRCA1 185delAG mutation is approximately 1 percent in Ashkenazi Jewish individuals. Nat Genet. 1995;11(2):198-200.

35. Elstrodt F, et al. BRCA1 mutation analysis of 41 
human breast cancer cell lines reveals three new deleterious mutants. Cancer Res. 2006;66(1):41-45.

36. Ignatoski KM, Ethier SP. Constitutive activation of pp125fak in newly isolated human breast cancer cell lines. Breast Cancer Res Treat. 1999;54(2):173-182.

37. Walsh $\mathrm{T}$, et al. Mutations in 12 genes for inherited ovarian, fallopian tube, and peritoneal carcinoma identified by massively parallel sequencing. Proc Natl Acad Sci U S A. 2011;108(44):18032-18037.

38. Buisson M, Anczuków O, Zetoune AB, Ware MD, Mazoyer S. The 185delAG mutation (c.68_69delAG) in the BRCA1 gene triggers translation reinitiation at a downstream AUG codon. Hum Mutat. 2006;27(10):1024-1029.

39. Gebauer F, Hentze MW. Molecular mechanisms of translational control. Nat Rev Mol Cell Biol. 2004;5(10):827-835.

40. Kozak M. Circumstances and mechanisms of inhibition of translation by secondary structure in eucaryotic mRNAs. Mol Cell Biol. 1989;9(11):5134-5142.

41. Kozak M. Constraints on reinitiation of translation in mammals. Nucleic Acids Res. 2001;29(24):5226-5232.

42. Hinnebusch AG. The scanning mechanism of eukaryotic translation initiation. Annu Rev Biochem. 2014;83:779-812.

43. Powley IR, et al. Translational reprogramming following UVB irradiation is mediated by DNAPKcs and allows selective recruitment to the polysomes of mRNAs encoding DNA repair enzymes. Genes Dev. 2009;23(10):1207-1220.

44. Joukov V, Chen J, Fox EA, Green JB, Livingston DM. Functional communication between endogenous BRCA1 and its partner, BARD1, during Xenopus laevis development. Proc Natl Acad Sci US A. 2001;98(21):12078-12083.

45. Woolery KT, Mohamed M, Linger RJ, Dobrinski KP, Roman J, Kruk PA. BRCA1 185delAG mutation enhances interleukin-1 $\beta$ expression in ovarian surface epithelial cells. Biomed Res Int. 2015;2015:652017.

46. O'Donnell JD, Linger RJ, Kruk PA. BRCA1 185delAG mutant protein, BRAt, up-regulates maspin in ovarian epithelial cells. Gynecol Oncol. 2010;116(2):262-268.

47. Perrin-Vidoz L, Sinilnikova OM, StoppaLyonnet D, Lenoir GM, Mazoyer S. The nonsense-mediated mRNA decay pathway triggers degradation of most BRCA1 mRNAs bearing premature termination codons. Hum Mol Genet. 2002;11(23):2805-2814.

48. Ceccaldi R, et al. A unique subset of epithelial ovarian cancers with platinum sensitivity and PARP inhibitor resistance. Cancer Res. 2015;75(4):628-634.

49. Spriggs KA, Bushell M, Willis AE. Translational regulation of gene expression during conditions of cell stress. Mol Cell. 2010;40(2):228-237.

50. Fabbro M, Rodriguez JA, Baer R, Henderson BR. BARD1 induces BRCA1 intranuclear foci for- mation by increasing RING-dependent BRCA1 nuclear import and inhibiting BRCA1 nuclear export. J Biol Chem. 2002;277(24):21315-21324.

51. Sobhian B, et al. RAP80 targets BRCA1 to specific ubiquitin structures at DNA damage sites. Science. 2007;316(5828):1198-1202.

52. Wang B, et al. Abraxas and RAP80 form a BRCA1 protein complex required for the DNA damage response. Science. 2007;316(5828):1194-1198.

53. Kim H, Chen J, Yu X. Ubiquitin-binding protein RAP80 mediates BRCA1-dependent DNA damage response. Science. 2007;316(5828):1202-1205.

54. Pathania $S$, et al. BRCA1 is required for postreplication repair after UV-induced DNA damage. Mol Cell. 2011;44(2):235-251.

55. Au WW, Henderson BR. The BRCA1 RING and BRCT domains cooperate in targeting BRCA1 to ionizing radiation-induced nuclear foci. J Biol Chem. 2005;280(8):6993-7001.

56. Chetrit A, Hirsh-Yechezkel G, Ben-David Y, Lubin F, Friedman E, Sadetzki S. Effect of BRCA1/2 mutations on long-term survival of patients with invasive ovarian cancer: the national Israeli study of ovarian cancer. JClin Oncol. 2008;26(1):20-25.

57. Seeger C, Sohn JA. Targeting Hepatitis B virus with CRISPR/Cas9. Mol Ther Nucleic Acids. 2014;3:e216.

58. Swisher EM, Sakai W, Karlan BY, Wurz K, Urban $\mathrm{N}$, Taniguchi T. Secondary BRCA1 mutations in BRCA1-mutated ovarian carcinomas with platinum resistance. Cancer Res. 2008;68(8):2581-2586. 